\title{
Damage identification for frame structures using vision-based measurement
}

\section{$\operatorname{AUTHOR}(\mathrm{S}):$}

Guo, Jia; Jiao, Jian; Fujita, Kohei; Takewaki, Izuru

\section{CITATION:}

Guo, Jia ...[et al]. Damage identification for frame structures using vision-based measurement. Engineering Structures 2019, 199: 109634.

\section{ISSUE DATE:}

2019-11-15

URL:

http://hdl.handle.net/2433/244209

\section{RIGHT:}

(C) 2019. This manuscript version is made available under the CC-BY-NC-ND 4.0 license

http://creativecommons.org/licenses/by-nc-nd/4.0/.; The full-text file will be made open to the public on 15 Novembe 2021 in accordance with publisher's 'Terms and Conditions for Self-Archiving'.; This is not the published version. Please cite only the published version.; この論文は出版社版でありません。引用の際には出版社版をご確認ご利用ください。 
1

2

3

4

5

6

7

\section{Introduction}

26 The main difficulty in localizing damages by means of model-based damage identification meth-

$\mathbf{2 7}$ ods with dynamic responses of structures, resides in that the number of the physical parameters

\footnotetext{
${ }^{*}$ Correspondence to: Jia Guo, Email: kaku.ka.25x@st.kyoto-u.ac.jp
}

\section{measurement}

Extracting physical parameters for damage identification problems from full-field measurements is a promising research because of the recent spread of vision-based measurement techniques in the experimental mechanics. This paper presents a vision-based measurement framework using the camera system for damage identification. The framework is composed of four procedures: camera calibration, image processing, system identification and sensitivity analysis. In contrast to traditional finite-point measurements, the camera system allows considerably greater non-contact measurement flexibility. Such flexibility has two imporproblems; and second, more physical parameters could be extracted, taking advantage of the plentiful experimental data. A laboratory test comparing the camera system to traditional accelerometer measurement is conducted to confirm the above advantages. Further statistic analysis shows that the major drawback of this technique is that the camera system presents high levels of noise in small vibration responses at higher frequencies. Suitable strategies to circumvent this disadvantage are developed. Moreover, a technique for practical camera calibration without the requirement that the objective plane should be strictly perpendicular to the camera axis is also demonstrated and verified by the proposed laboratory test.

Keywords: vision-based measurement, damage identification, sensitivity analysis, homography estimation 
1 to be recovered is by far larger than the number of measurements. This always makes the

2 identification problem underdetermined with infinite number of possible solutions, or typically

3 ill-posed. In practice, using more than one set of measurements could improve the identifiability

4 and robustness of the identification procedure and thus provide a remedy for the possible uniden-

5 tifiability of the identification approach [1]. However, a large number of tests can be extremely

6 time and labor consuming and are sometimes not easy to be conducted in tough environments

7 or for in-situ measurements. The recently spread vision-based full-field vibration measurement

8 techniques and the improvements in image processing offer potentials to achieve an important

9 breakthrough for this problem $[2,3]$. Compared to traditional finite-point measurement tech-

10 niques, full-field vibration measurements, normally by using industrial or consumer-grade video

11 cameras, allow to extract more information from small numbers of tests and thus more phys-

12 ical parameters may be identified. Superiority of the camera system also includes its flexible

13 measurement distance and commonplace availability [4-6].

14 The process of the vision-based vibration measurements using a camera system mainly in-

15 volves two key steps: camera calibration and image processing. Camera calibration is the

16 process of determining the intrinsic and extrinsic parameters of the camera setup and it allows

17 the camera system to determine a relationship between what appears on an image and where

18 it is located in the world [7]. The scale factor approach $[8,9]$ has been widely used to convert

19 the image coordinates measured by a camera system into space coordinates with the require-

20 ment that the objective plane should be perpendicular to the camera axis. For more general

21 cases, many calibration methods are proposed based on planar homography [10, 11]. However,

22 research shows that result accuracy of intrinsic parameters depends greatly on homography es-

23 timation and thus it is sensitive to noise [12]. An accurate and efficient numerical method for

24 homography estimation should be carefully chosen for the success of vision-based measurement.

25 After camera calibration, image processing could be adopted. In vibration measurements, the

26 objective of image processing is mainly to extract structural motions from recorded successive

27 images. Three methods are commonly used: point-tracking, digital image correlation (DIC), and

28 target-less approaches [13]. The DIC [14, 15] works based on gray-scale variations of continuous

29 patterns. The performance of DIC is greatly affected by the quality of speckle patterns $[16,17]$.

30 As regards the point tracking technique, it uses cameras to identify the coordinates of discrete

31 points (markers) mounted to test structures and the only requirement of the markers used in

32 the point-tracking technique is that they should have high contrast or be retro reflective, which

33 makes this technique much easier to be applied for field measurements [9]. 
1 Despite extensive efforts being made towards reliable and accurate digital data acquisition 2 and system identification [18-20], the study of practical vision-based applications for damage 3 identification is still limited. The methods using dynamic response data in the damage identi-

4 fication process can be simply classified into two groups: methods based on local singularity in

5 signals and methods based on finite element model updating. Singularity based methods (e.g., 6 wavelet analysis [21], Fourier spectral-based modal curvature analysis [22], etc.) received much 7 attention in recent years for the capability to avoid modeling errors and high computational 8 cost by directly detecting the discontinuities and breakdown points of measurement data, while 9 the methods based on finite element model updating identify damages by comparing the dis10 crepancy between analytical and experimental models and modifying the corresponding physical 11 parameters until the correlation of analytical model and experimetnal results satisfies practical 12 requirements [23]. In this research, the sensitivity method in finite element model updating 13 [24] is used for damage identification for its ability to identify the damage location and severity 14 simultaneously and capability to take into account all of the available structural information. It 15 should be noted that there is no constraint to apply the vision-based measurements into both the 16 singularity-based method as well as the finite element model updating method, especially that 17 the access to full-field displacement data accommodates the modal curvature analysis or other 18 derivatives based analysis in a very natural way. Research using full-field vibration measurement 19 data in damage identification can be referred to [25-27].

20 Problems rise by using the vision-based measurement for damage identification, for the reason 21 that identification of parameters using large sets of measured data requires especially careful 22 calibration processes and suitable computational strategies. Inaccurate frame rate and noisy 23 measurement data at higher frequencies might have a pronounced effect on the final identifica24 tion results yet get side stepped by the preliminary research. Moreover, the overall advantages 25 of using rich vibration measurement data in damage identification have not been clarified. Fi26 nally, many of the vision-based measurement techniques are still limited to laboratory tests, 27 which are not suitable for practical damage identification. The objective of this paper is to 28 present experimental investigations and comparative studies on the synthetic research of vision29 based displacement measurement obtained by the camera system and damage identification. In 30 contrast to traditional finite-point measurements, the camera system is equipped as features ex31 tracted from images that allow considerably greater non-contact measurement flexibility. Such 32 flexibility has two important benefits: first, less number of modes is required for modal-based 33 damage identification problems; and second, the information of large numbers of physical pa- 
1 rameters could be extracted, taking advantage of the plentiful experimental data. The chosen

2 identified parameters are most often associated with constitutive properties of the test sample

3 material and should be sensitive to changes in the measurement data. Detailed study that in-

4 cludes several strategies of different parameterisations is described in [28]. A laboratory test

5 comparing the camera system to the accelerometer measurement is carried out on a plane frame

6 structure to confirm the above advantages of the vision-based measurement technique. Based

7 on the statistic analysis of the laboratory test, a major drawback of this technique has also been

8 found that the camera system presents high levels of noise in small vibration responses at higher

9 frequency range. The effect of the uncertainties of the corresponding identified modal proper-

10 ties on the accuracy of structural damage identification is investigated. Suitable computational

11 strategies by choosing the optimal weights for the sensitivity-based damage identification method

12 to circumvent this disadvantage are proposed. Furthermore, a technique for practical camera

13 calibration without the requirement that the objective plane should be strictly perpendicular to

14 the camera axis is also demonstrated and verified by the proposed laboratory test.

\section{Vision-based measurement system}

\section{$16 \quad 2.1$ Camera calibration}

17 Camera calibration is the process of estimating parameters of the camera system so as to measure

18 structural responses from the captured video images. To translate the image coordinates (pixels)

19 into space coordinates (meters), the scaling factor approach is widely employed in previous

20 research for two-dimensional (2D) displacement measurements. However, the prerequisite of this

21 approach is the perpendicularity of the axis of camera to the object plane. It is shown that the

22 performance of the scale factor approach deteriorates greatly when the angle of inclination occurs

23 [10]. Making the object plane strictly perpendicular to the camera axis is difficult for practical

24 applications. To solve this problem, the direct linear transformation (DLT) for homography

25 estimation is introduced herein.

26 A homography maps from $P^{2} \rightarrow P^{2}$ by using a non-singular $3 \times 3$ homogenous matrix $\boldsymbol{H}$

27 with 8 degrees of freedom such that for any point in $P^{2}$ represented by vector $\boldsymbol{x}$, its mapped

28 point $\boldsymbol{x}^{\prime}$ equals $\boldsymbol{H} \boldsymbol{x}$. There are many kinds of methods for homography estimation, where the

29 DLT algorithm is the most practical and convenient one due to its linearity and simplicity. In 
1 homogenous coordinates, the relationship between $\boldsymbol{x}$ and $\boldsymbol{x}^{\prime}$ could be expressed as

$$
\boldsymbol{x}^{\prime}=\left(\begin{array}{l}
u \\
v \\
1
\end{array}\right)=\left(\begin{array}{lll}
h_{11} & h_{12} & h_{13} \\
h_{21} & h_{22} & h_{23} \\
h_{31} & h_{32} & h_{33}
\end{array}\right)\left(\begin{array}{l}
x \\
y \\
1
\end{array}\right)=\boldsymbol{H} \boldsymbol{x}
$$

2 Dividing the first row of Eq. (1) by the third row and the second row by the third row one 3 obtains:

$$
\boldsymbol{A}_{i} \boldsymbol{h}=\mathbf{0}
$$

4 where

$$
\begin{aligned}
& \boldsymbol{A}_{i}=\left(\begin{array}{ccccccccc}
-x & -y & -1 & 0 & 0 & 0 & u x & u y & u \\
0 & 0 & 0 & -x & -y & -1 & v x & v y & v
\end{array}\right) \\
& \boldsymbol{h}=\left(\begin{array}{lllllllll}
h_{11} & h_{12} & h_{13} & h_{21} & h_{22} & h_{23} & h_{31} & h_{32} & h_{33}
\end{array}\right)^{\mathrm{T}}
\end{aligned}
$$

5 Since each pair of coordinates for one calibration point provides 2 equations, 4 points are suf-

6 ficient to solve for the 8 degrees of freedom of $\boldsymbol{H}$. The restriction is that no 3 points can be

7 collinear. In practice, a better solution of $\boldsymbol{h}$ could be obtained by more than 4 calibration points.

8 The problem then becomes to solve for a vector $\boldsymbol{h}$ that minimizes a suitable cost function. More-

9 over, as the DLT algorithm is dependent on the origin and scale of the coordinate system in

10 the image, a normalization step is required to ensure that the solution converges to the correct

11 result. For comprehensive study about this algorithm, the reader is referred to [29].

12 The following table is used to show the above camera calibration procedure more clearly.

- Identify the calibration point correspondences $\left(\boldsymbol{x}, \boldsymbol{x}^{\prime}\right)$ between the image plane and the measured object plane.

- Normalize the correspondences $\left(\boldsymbol{x}, \boldsymbol{x}^{\prime}\right)$ to $\left(\tilde{\boldsymbol{x}}, \tilde{\boldsymbol{x}}^{\prime}\right)$ so as to ensure the solution coverges to the correct result [7].

- Apply the DLT algorithm using $\tilde{\boldsymbol{x}}$ and $\tilde{\boldsymbol{x}}^{\prime}$ to obtain homography matrix $\tilde{\boldsymbol{H}}$.

- Get the final homography matrix $\boldsymbol{H}$ considering the normalization step.

- Apply the above homography matrix $\boldsymbol{H}$ to every frame of the given video.

13 An example of using the homography estimation from images by the DLT algorithm for 14 camera calibration is shown in Figure 1. In this example, the calibration points were acquired 15 by installing a calibration board. In the following laboratory test, however, six known-position 16 reference markers were utilized as the calibration points for the reason that these six reference 


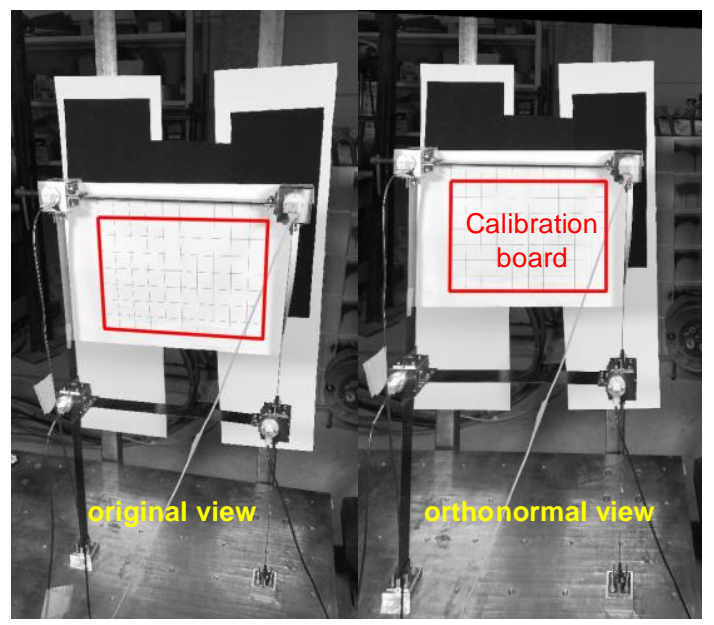

(a)

(b)

Figure 1 Example of homography estimation: (a) before calibration; (b) after calibration

1 markers and the other markers defined the measured object plane. This procedure will be 2 described in detail in Section 4.

\section{$\begin{array}{lll}3 & 2.2 & \text { Image processing }\end{array}$}

4 After the construction of the camera calibration matrix $\boldsymbol{H}$, image processing could be adopted

5 by a commercial software. In this research, image processing refers to converting an image

6 into digital form in order to extract the motions of the structure by point tracking method.

7 Natural features or artificial markers with high contrast in the object plane can be selected as

8 the tracked points for motion extraction (Figure 2(a)). Then correlation trackers are assigned

9 to the selected points. After the trackers are set up, the correlation tracking function is used so

10 as to look in each successive image for the part of the image contained within the correlation

11 circle (the purple template in Figure 2(b)). Tracker tolerance should be set carefully as it is a

12 threshold that defines if a point is to be considered lost or not. Set a higher value to increase

13 the tolerance, e.g. in a situation where the tracker is producing accurate results, but the noise

14 level in the images causes them to be rejected because they are outside the default tolerance.

15 Tracking results from all images are recorded as the space coordinates of the tracked points and

16 physical displacements could be determined accordingly.

17 The aforementioned vision-based measurement procedures (sections 2.1 and 2.2) are sum-

18 marized in Figure 3. 


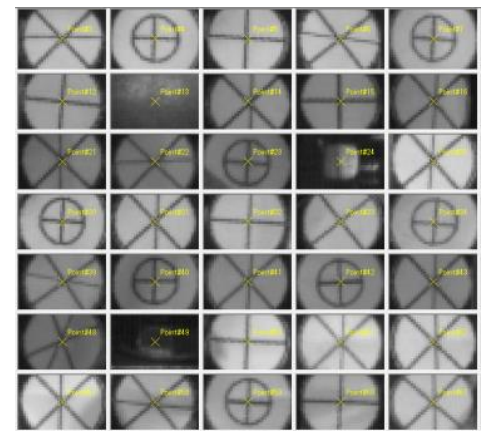

(a)

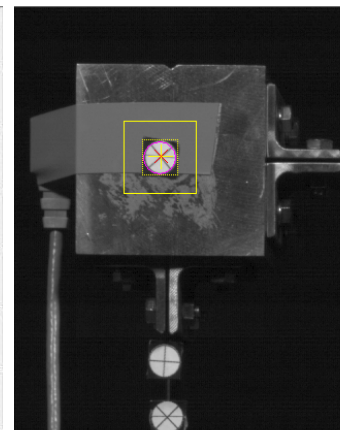

(b)

Figure 2 Markers for image processing: (a) different types of markers used in the laboratory test; (b) correlation circle of a marker

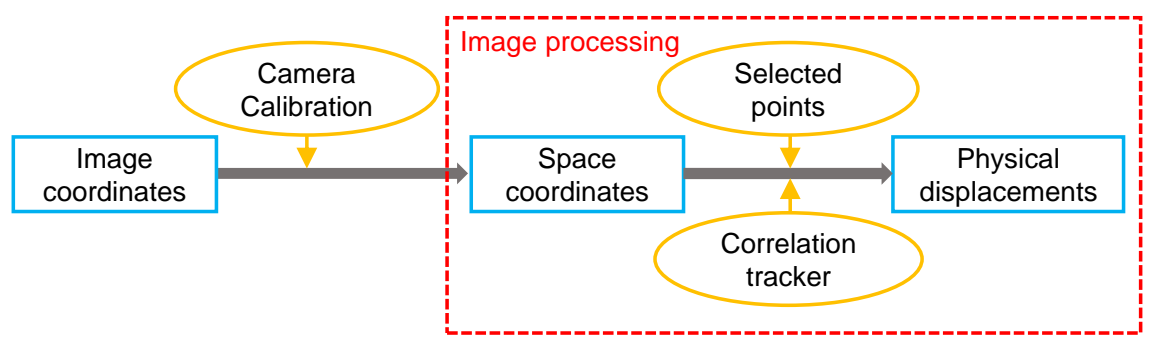

Figure 3 Overview of the vision-based measurement system

\section{Sensitivity analysis for damage identification}

2 Damage identification is an inverse problem of finding the appropriate unknown physical pa-

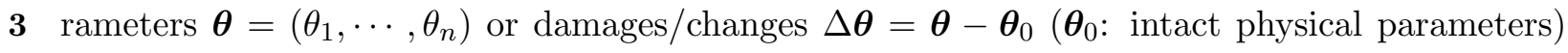

4 from a set of observation data. This process is usually formulated as a nonlinear optimization

5 problem whose objective function is the weighted least squares of the error between measured

6 data $\hat{\boldsymbol{R}}$ and the corresponding analytically predicted data $\boldsymbol{R}(\boldsymbol{\theta})$, i.e.,

$$
\Delta \boldsymbol{\theta}^{*}=\arg \min g(\Delta \boldsymbol{\theta}):=\left\|\hat{\boldsymbol{R}}-\boldsymbol{R}\left(\boldsymbol{\theta}_{0}+\Delta \boldsymbol{\theta}\right)\right\|_{\boldsymbol{W}}^{2}
$$

$\mathbf{7}$ where $\boldsymbol{\Delta} \boldsymbol{\theta}^{*}$ are the identified damages and $\boldsymbol{W}=\operatorname{diag}\left(\left[\boldsymbol{W}_{1}, \cdots, \boldsymbol{W}_{l}\right]\right)$ is the positive definite 8 weight matrix. Eq. (4) is the basic expression for the above nonlinear optimization problem.

9 Practically, regularization terms, namely Tikhonov regularization [30, 31] and sparse regular-

10 ization $[32,33]$, are always additionally introduced to allow a robust approximation of the final

11 result. In this research, Tikhonov regularization is used, for the convenience and effectiveness

12 of its application. The change vector of $\hat{\boldsymbol{R}}-\boldsymbol{R}\left(\boldsymbol{\theta}_{0}+\Delta \boldsymbol{\theta}\right)$ can be obtained iteratively by the

13 sensitivity method [23, 24]. In the sensitivity method, a truncated Taylor series expansion of

14 the predicted data $\boldsymbol{R}(\boldsymbol{\theta})$ in terms of the damage parameters $\boldsymbol{\theta}$ at given $\overline{\boldsymbol{\theta}}$ is used, which leads to 
1 the linear approximation of the original nonlinear optimization problem, as shown in Eq. (5):

$$
\hat{\boldsymbol{R}}-\boldsymbol{R}(\boldsymbol{\theta})=\Delta \boldsymbol{R}(\overline{\boldsymbol{\theta}})-\boldsymbol{S}(\overline{\boldsymbol{\theta}}) \Delta \boldsymbol{\theta}
$$

2 To this end, sensitivity analysis should be involved so as to get the sensitivity matrix $\boldsymbol{S}(\overline{\boldsymbol{\theta}})$.

$$
\boldsymbol{S}(\overline{\boldsymbol{\theta}})=\nabla \boldsymbol{R}(\overline{\boldsymbol{\theta}}):=\left[\frac{\partial \boldsymbol{R}}{\partial \theta_{1}}, \frac{\partial \boldsymbol{R}}{\partial \theta_{2}}, \cdots, \frac{\partial \boldsymbol{R}}{\partial \theta_{m}}\right]
$$

3 It is noteworthy that for different types of measured data $\hat{\boldsymbol{R}}$, the sensitivity matrix $\boldsymbol{S}$ is derived

4 differently. In this research, measured data $\hat{\boldsymbol{R}}$ refer to the vision-based identified structural

5 natural frequencies and mode shapes. The basic objective function combining frequency changes

6 and mode shape changes for damage identification takes the form

$$
g(\boldsymbol{\theta})=\sum_{j=1}^{m} W_{\lambda_{j}}^{2}\left(\frac{\hat{\lambda}_{j}-\lambda_{j}\left(\boldsymbol{\theta}_{0}+\Delta \boldsymbol{\theta}\right)}{\hat{\lambda}_{j}}\right)^{2}+\sum_{j=1}^{m} W_{\phi_{j}}^{2} \sum_{i=1}^{n p}\left(\hat{\phi}_{j i}-\phi_{j i}\left(\boldsymbol{\theta}_{0}+\Delta \boldsymbol{\theta}\right)\right)^{2}
$$

7 where $\lambda_{j}$ is the $j$ th eigenvalue and $\left\{\phi_{j i}\right\}$ is the $i$ th component of the $j$ th normalized mode shape

$8 \phi_{j}$. The sensitivity of the $j$ th eigenvalue $\lambda_{j}$ and the corresponding mode shape $\phi_{j}$ to the damage

9 parameter $\theta_{k}$ is formulated as

$$
\begin{aligned}
& \frac{\partial \lambda_{j}}{\partial \theta_{k}}=\boldsymbol{\phi}_{j}^{\mathrm{T}}\left(-\lambda_{j} \frac{\partial \boldsymbol{M}}{\partial \theta_{k}}+\frac{\partial \boldsymbol{K}}{\partial \theta_{k}}\right) \boldsymbol{\phi}_{j} \\
& \frac{\partial \boldsymbol{\phi}_{j}}{\partial \theta_{k}}=\boldsymbol{v}_{j}+c_{j} \boldsymbol{\phi}_{j}
\end{aligned}
$$

10 where $\boldsymbol{M}, \boldsymbol{K} \in \mathbb{R}^{n \times n}$ are the mass and stiffness matrices, respectively. The mode shape deriva-

11 tive is given in two parts $\boldsymbol{v}_{j}$ and $c_{j} \phi_{j}$ :

$$
\begin{aligned}
& \boldsymbol{v}_{j}=\left[\overline{\boldsymbol{K}}-\lambda_{j} \overline{\boldsymbol{M}}\right]^{-1} \overline{\boldsymbol{f}}, \boldsymbol{f}=-\left[\frac{\partial \boldsymbol{K}}{\partial \theta_{k}}-\lambda_{j} \frac{\partial \boldsymbol{M}}{\partial \theta_{k}}-\boldsymbol{\phi}_{j}^{\mathrm{T}}\left[\frac{\partial \boldsymbol{K}}{\partial \theta_{k}}-\lambda_{j} \frac{\partial \boldsymbol{M}}{\partial \theta_{k}}\right] \boldsymbol{\phi}_{j} \boldsymbol{M}\right] \boldsymbol{\phi}_{j} \\
& c_{j}=-\boldsymbol{\phi}_{j}^{\mathrm{T}} \boldsymbol{M} \boldsymbol{v}_{j}-\frac{1}{2} \boldsymbol{\phi}_{j}^{\mathrm{T}} \frac{\partial \boldsymbol{M}}{\partial \theta_{k}} \boldsymbol{\phi}_{j} .
\end{aligned}
$$

12 Suppose the $j$ th mode shape $\left|\left\{\phi_{j i}\right\}\right|$ reaches the maximum value at the location $r, \overline{\boldsymbol{K}}, \overline{\boldsymbol{M}} \in$ $13 \mathbb{R}^{(n-1) \times(n-1)}$ are extracted from $\boldsymbol{M}$ and $\boldsymbol{K}$ by eliminating the $r$ th row and the $r$ th column.

$14 \overline{\boldsymbol{f}} \in \mathbb{R}^{n-1}$ is obtained by eliminating the $r$ th term of $\overline{\boldsymbol{f}}$. The reason for this elimination is

15 that rank of $\boldsymbol{K}-\lambda_{j} \boldsymbol{M}$ equals to $n-1$ and the $r$ th equation should be strongly coupled to the

16 redundancy and can therefore be removed [34].

\section{Laboratory test of a two-story plane frame structure}

\section{$18 \quad 4.1 \quad$ Test setup}

19 To validate the effectiveness of the proposed vision-based technique combined with its application

20 to damage identification, a two-story frame structure was designed as the analyzed structure to

21 be tested, as shown in Figure 4. In this test, there are four main systems to be setup: 


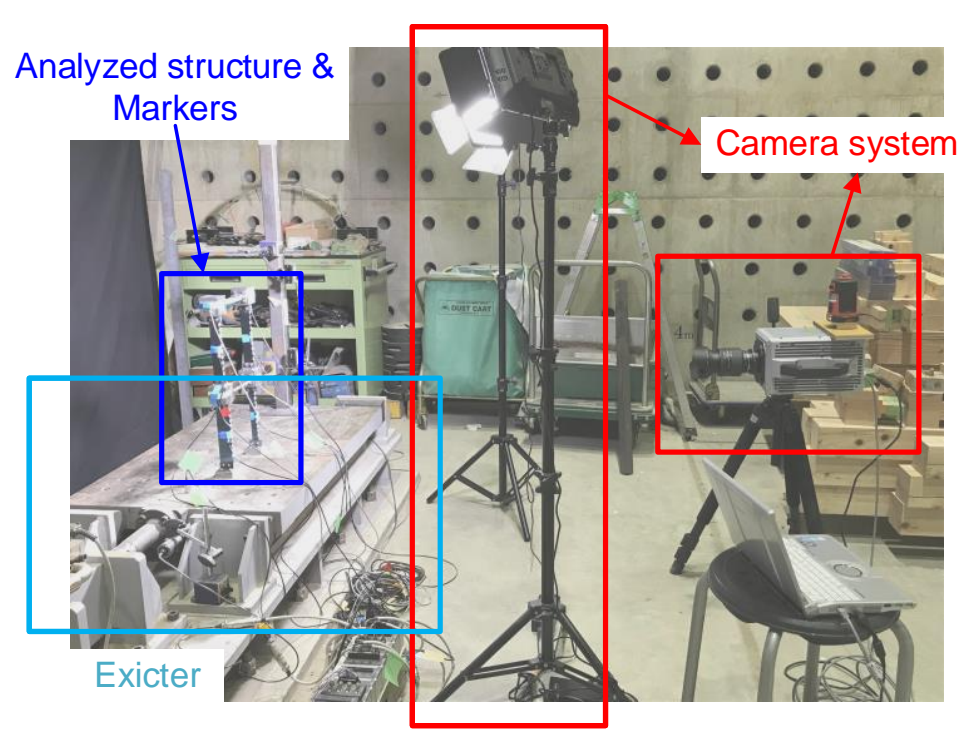

Figure 4 Four main systems

1. Camera system: One high-speed camera (model FASTCAM SA6 75K-M3IT) with a full resolution of $1920 \times 1440$ pixels and aperture set at $\mathrm{f} / 8$ is used to record the in-plane dynamic behaviors of the analyzed structure and the seismic table. The camera shutter time is $1 / 1000$ s. For this test, full resolution images were used with a frame rate of 500 fps. The camera system uses $72 \mathrm{~mm}$ lenses with focal length of $24-85 \mathrm{~mm}$ and field angle of $33.4^{\circ}$. In order to improve the accuracy of the measurement system, a black background and two $260 \times 188 \mathrm{~mm}^{2}$ LED light panels with the power of 4680 lumens were used. The video images captured by the camera were streamed into the camera onboard memory (64GB) first through an Ethernet cable and then saved into the computer. Structural displacements were obtained by the commercial software TEMA 4.0.

2. Analyzed structure: Figure $\mathbf{5}$ shows the details of the selected plane frame structure. Parameters of this frame are: height of the first story $H_{1}=0.225 \mathrm{~m}$, height of the second story $\mathrm{H}_{2}=0.25 \mathrm{~m}$, width $B=0.2 \mathrm{~m}$, rectangular cross-section of the first story's beam/column $b_{1} \times h_{1}=40 \mathrm{~mm} \times 1 \mathrm{~mm}$, rectangular cross-section of the second story's beam/column $b_{2} \times h_{2}=30 \mathrm{~mm} \times 1 \mathrm{~mm}$, lumped mass pair of the first story $m_{1}=1.16 \mathrm{~kg}$, lumped mass pair of the second story $m_{2}=1.09 \mathrm{~kg}$. Young's modulus and mass density of the beam/column are estimated as $E=200 \mathrm{GPa}, \rho=7.7 \times 10^{3} \mathrm{~kg} / \mathrm{m}^{3}$. The size of the lumped mass is measured as $50 \mathrm{~mm} \times 50 \mathrm{~mm} \times 50 \mathrm{~mm}$. Lumped mass and beam/column are connected by L-angles with size $40 \mathrm{~mm} \times 20 \mathrm{~mm} \times 2 \mathrm{~mm}$. Damage is introduced by reducing half of the width of the rectangular cross section in the area of left $1 / 2$ length of the beam at the first story $\left(b_{1}^{\prime}=20 \mathrm{~mm}\right)$. As a result, the approximated stiffness reduction 


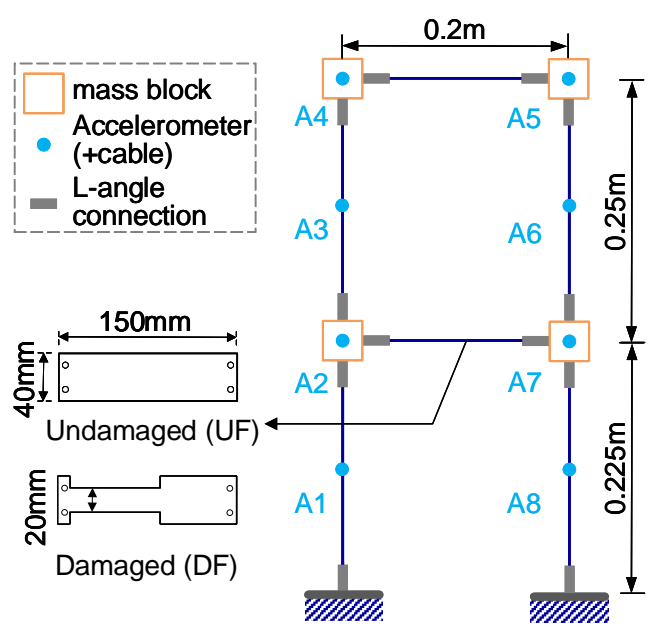

Figure 5 Overview of the analyzed structure

1

$\left(k^{\text {damage }} / k_{0}=b_{1}^{\prime} / b_{1}\right.$, where $k=E I$ is the bending stiffness and $I=b h^{3} / 12$ is the second moment of inertia of beam cross-section) for this area is estimated as $50 \%$.

3. Exciter: A series of harmonic excitations at natural frequencies $2.7 \mathrm{~Hz}$ (mode 1), $8.0 \mathrm{~Hz}$ (mode 2), $46.2 \mathrm{~Hz}$ (mode 4) and $53.2 \mathrm{~Hz}$ (mode 5) for the analyzed structure were provided by the seismic table in the structure laboratory in Kyoto University. Harmonic excitations at each frequency were repeated 4-8 times. Between the repeats at the same excitation frequency, small differences could be observed from the following system identification results and the mean values as well as the standard deviations of the structural natural frequencies and mode shapes were obtained consequently, which is discussed in detail in section 4.3. The analyzed structure was bolted on the seismic table. Stainless L-angle connection was used for the column base connection. Displacement of the seismic table was also captured by the camera system.

4. Markers: 65 dense markers were used in the test as shown in Figure 6, including 63 artificial markers (T1-T63) glued to the object plane and 2 bolt points (B1-B2) being selected due to their natural high-contrast features. Among all of the markers, six markers (yellow circles in Figure 6) were utilized as reference markers for camera calibration with accurate measurement of their physical dimension on the object plane. Through those six known-position points, one can obtain the camera calibration matrix $\boldsymbol{H}$ and thus translate the image coordinates into space coordinates for any point in the objective plane. 


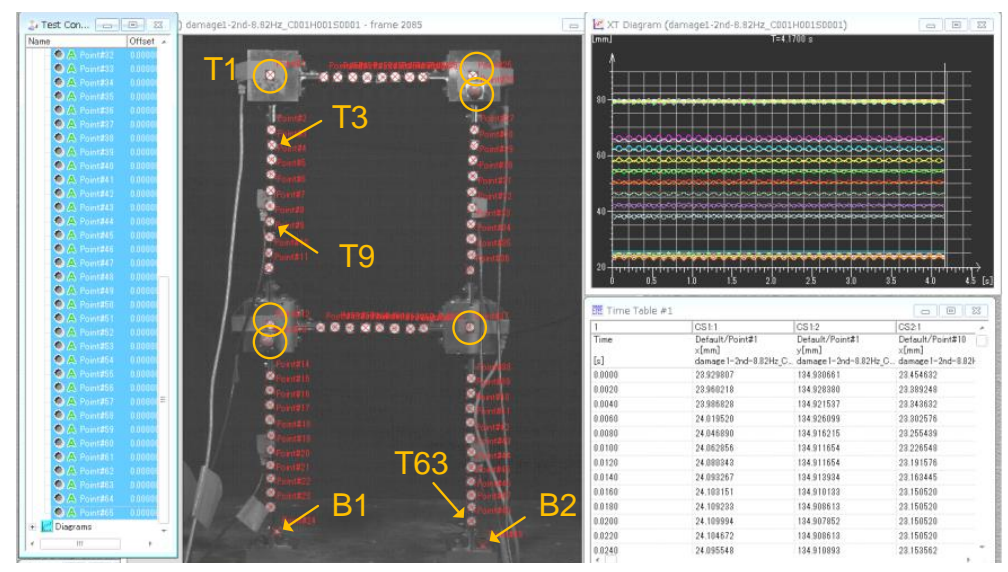

Figure 6 User interface of TEMA Motion Analysis Software and markers setup

Table 1 Measured frequencies with coefficients of variation

\begin{tabular}{lccccccc}
\hline \multirow{2}{*}{ Measurement } & \multirow{6}{*}{ Frequency } & \multicolumn{7}{c}{ Mode } \\
\cline { 3 - 8 } & & 1 & 2 & 3 & 4 & 5 & 6 \\
\hline \multirow{2}{*}{ Vision-based } & Mean $(\mathrm{Hz})$ & 2.64 & 7.97 & - & 45.49 & 53.13 & - \\
& $\mathrm{COV}\left(\times 10^{-2}\right)$ & 0.13 & 0.18 & - & 0.21 & 0.20 & - \\
\multirow{3}{*}{ Accelerometer } & Mean $(\mathrm{Hz})$ & 2.65 & 8.02 & 26.56 & - & 53.27 & 67.36 \\
& $\mathrm{COV}\left(\times 10^{-2}\right)$ & 0.02 & 0.04 & 0.07 & - & 0.05 & 0.20 \\
\hline
\end{tabular}

\section{$1 \quad 4.2$ Displacement results}

2 Steady-state displacements from two markers (T3 and T9 in Figure 6) of the analyzed structure 3 at harmonic excitation $53.2 \mathrm{~Hz}$ are illustrated in Figure 7 (a)-(b). Compared with marker T3, 4 a systematic data shift due to the sensor drift could be clearly found in marker T9. This 5 phenomenon happened during the marker tracking and it is obviously not meaningful. As a 6 result, a linear detrending process was conducted. The displacement responses of T3 and T9 7 after detrending are shown in Figure 7 (c)-(d).

8 For higher modes of the analyzed structure, the frame was weakly excited (amplitude $9 \approx 0.5 \mathrm{~mm}$ in Figure 7 ). To eliminate unwanted noises so as to make the following system 10 identification results more robust, filtered displacement responses were obtained by the appli-

11 cation of a band pass filter with a passband frequency 40-50 Hz (mode 4) and 50-60 $\mathrm{Hz}$ (mode 12 5), as shown in Figure 7 ((e)-(f)). Based on the filtered displacement responses, the structural 13 dynamic responses around the harmonic excitation frequency could be clearly identified. 

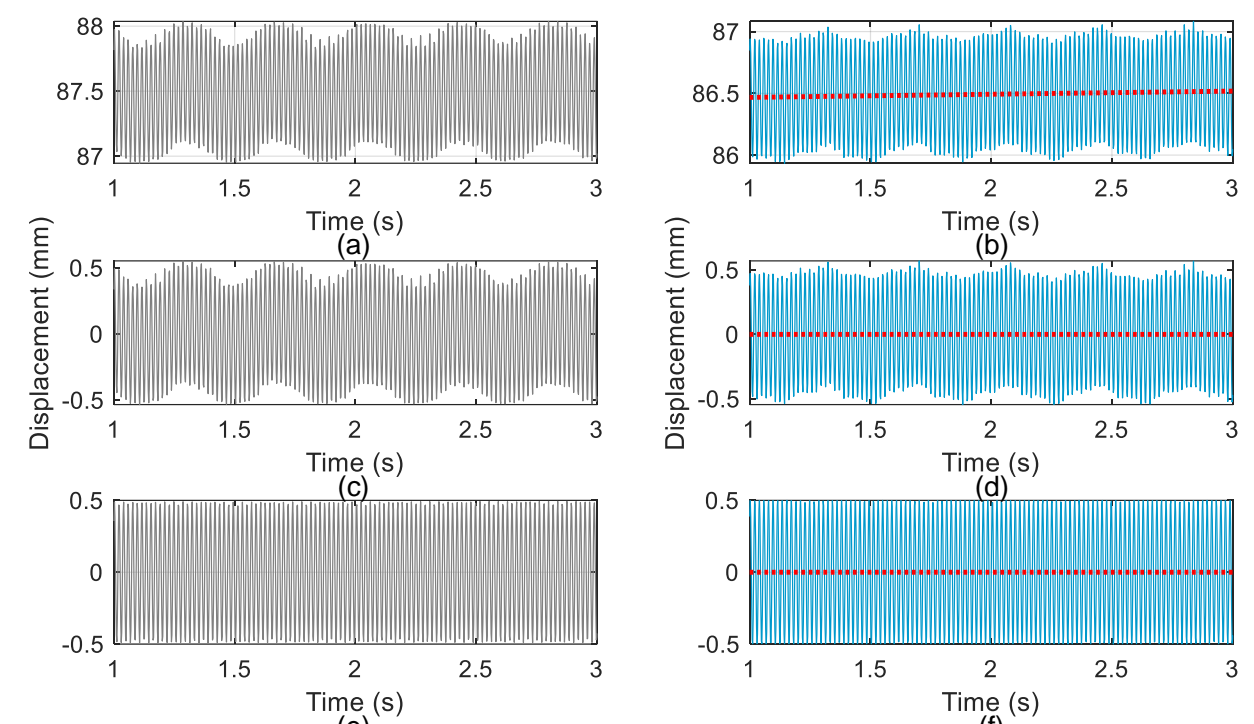

(e)

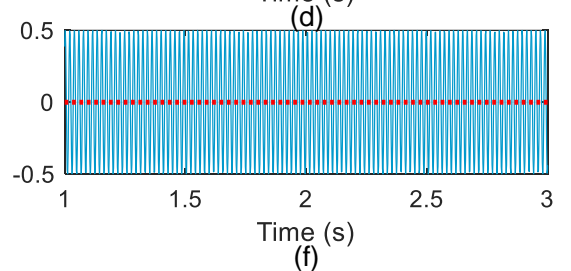

Figure 7 Steady-state displacements at $53 \mathrm{~Hz}$ : (a) original signal of T3; (b) original signal of T9; (c) T3 after detrending; (d) T9 after detrending; (e) T3 after filtering; (f) T9 after filtering

\section{$1 \quad 4.3 \quad$ System identification results}

2 Natural frequencies and mode shapes of the analyzed structure were then determined by the

3 subspace identification algorithm. The mean value of identified natural frequencies with coef-

4 ficients of variation (COV) over several repeated tests are listed in Table 1, comparing with

5 the results obtained by accelerometers. The mean and mean \pm one standard deviation of the

6 identified mode shapes are additionally depicted in Figure 8, where mass normalization for

7 mode shapes was used.

8 From these statistics properties of the modal data, several conclusions can be made. (1) The

9 accelerometer achieved more accurate results than the vision-based measurement in regard to

10 the COV of the natural frequencies in Table 1. The mean value of the vision-based frequencies

11 is also subject to bias errors. This is because the vision-based measurement had shorter mea-

12 surement duration than the accelerometer due to the camera's limited onboard memory. (2) For

13 mode shape measurement, the camera system presents high levels of noise in small vibration re-

14 sponses at higher frequencies than traditional accelerometers. One can find that the vision-based

15 measurement failed to identify mode 3 of the analyzed structure and although modes 4-5 were

16 successfully identified, they had quite high standard deviations in the identified mode shapes (as

17 shown in Figure 8). The reason behind this phenomenon is that due to the control limitation of

18 the operating area of the seismic table, the higher the excitation frequency is applied, the lower 


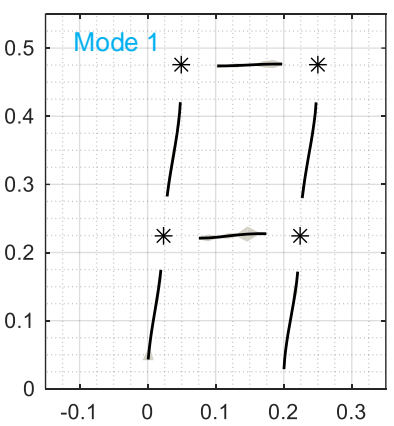

(a)

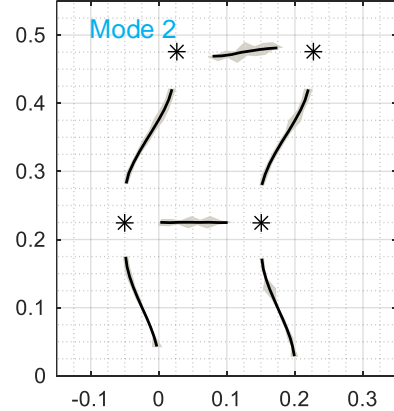

(b)

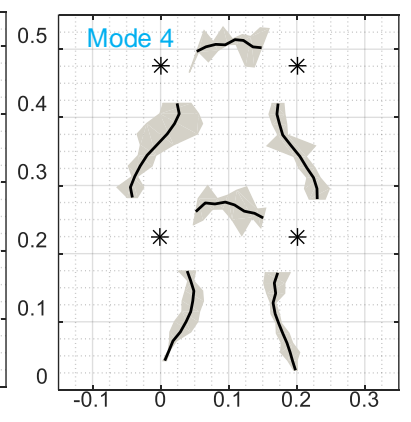

(c)

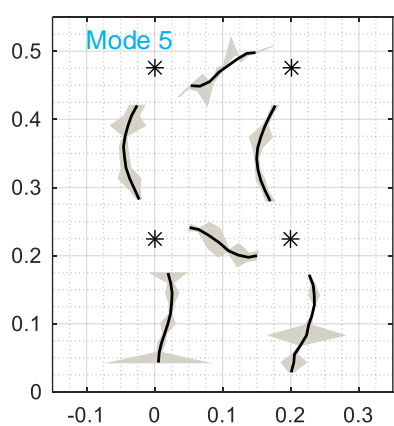

(d)

Figure 8 Mean (black) and mean $\pm 20 \times$ standard deviation (gray) of identified mode shapes by vision-based measurement

1 displacement amplitude can be obtained for the seismic table. Thus structural displacement re-

2 sponses of these modes are hard to be caught. It is well known that the displacement amplitude

3 of the higher mode for practical structures is also very small. Using accelerometers could attain

4 better results since accelerations are more sensitive to higher frequencies than displacements.

5 Awareness of these drawbacks is important because special attentions should be paid in the

6 following damage identification process.

\section{$7 \quad 4.4 \quad$ Damage identification}

8 The numerical baseline model is established according to the geometric and physical properties

9 of the analyzed structure with 85 elements and 85 nodes, as shown in Figure 9(c). Each node

10 has three degrees of freedom (DOFs) and the model has 255 DOFs in total. Among all of the

11 elements, elements 1-63 refer to the possibly damaged beam/column elements where unknown

12 damage parameters $\boldsymbol{\theta}=\left(\theta_{1}, \cdots, \theta_{63}\right)$ are assigned to, while elements 64-85 refer to the lumped

13 mass and L-angle parts with known stiffness value. The initial values of the damage parameters

$14 \boldsymbol{\theta}$ are set to be 1 (stiffness reduction set to be zero), assuming no damage for the structure

15 before damage identification. The desired identified values of the damage parameters should be

$16\left(\theta_{12}, \theta_{13}, \theta_{14}, \theta_{15}\right)=0.5$ and the values for other $\theta$ should not deviate from the initial value.

17 Only modal data of modes 1-2 and 4-5 were used for damage identification. The mean values

18 of identified mode shapes $\left(\hat{\phi}_{j i}\right)$ in Figure 8, the mean values of vision-based identified natural

19 frequency of mode 4 and accelerometer identified natural frequencies of mode 1,2 and 5 were

20 being set as input in Eq. (7) $\left(\hat{\lambda}_{j}=\left(2 \pi \hat{f}_{j}\right)^{2}\right)$. The reason why some accelerometer identified

21 frequencies were needed is that the statistics analysis in Table $\mathbf{1}$ shows that the data from

22 accelerometers achieved much more accurate system identification results in terms of natural 

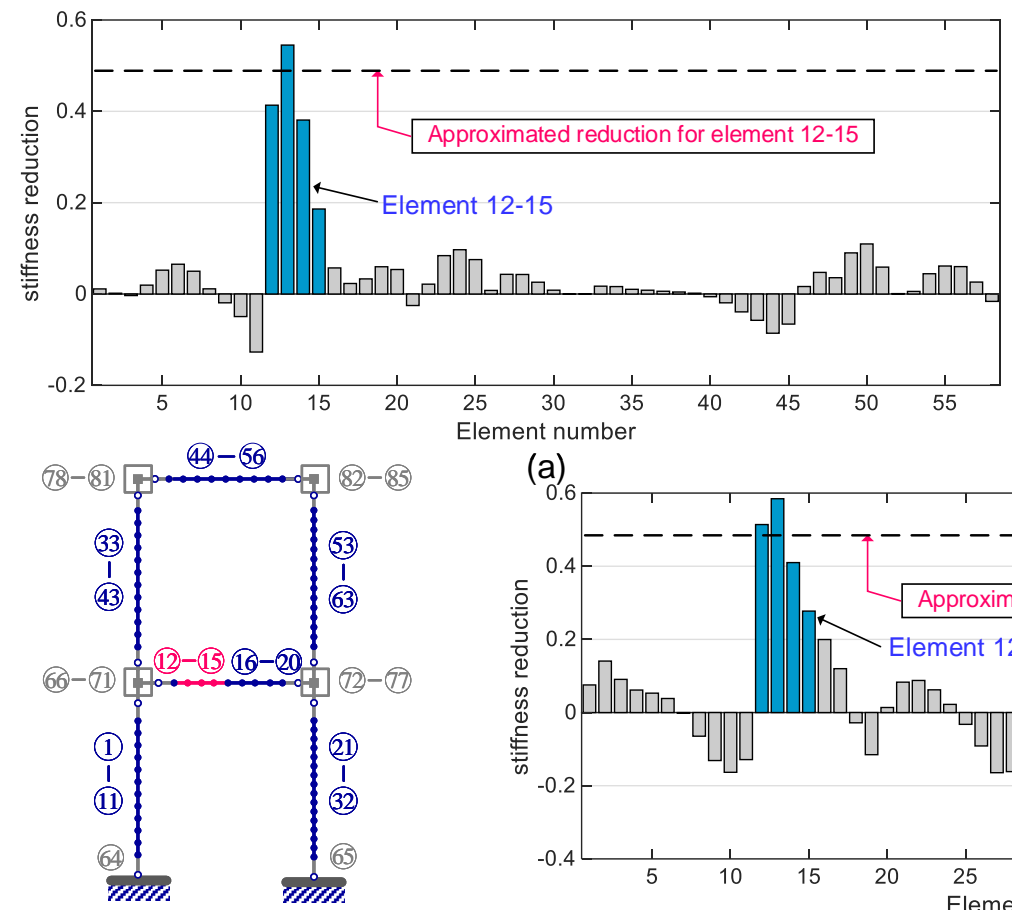

(c)

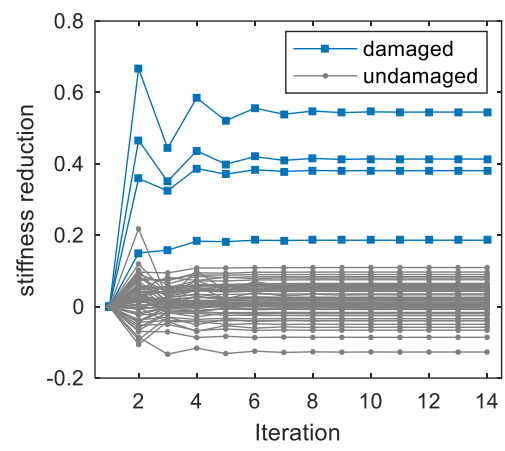

(b)

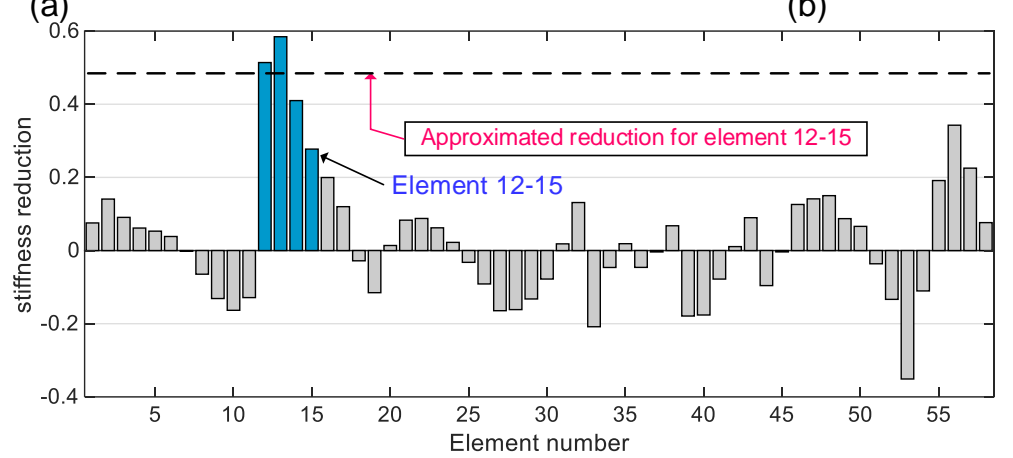

(d)

Figure 9 Identification results using vision-based measurements: (a) with optimal weights; (b) history of convergence; (c) numerical model; (d) with identity weights

1 frequencies. As the identification of large numbers of unknown physical parameters is more

2 sensitive to the measurement noise, it is safe to directly use these more accurate data. Careful

3 verifications should be conducted if only the vision-based identified natural frequencies are being

4 used, see Section 5.1 for more details. The final damage identification results are shown in

5 Figure 9(a)-(b). Figure 9(a) shows that nearly all of the elements 12-15 are detected as having

6 damages close to $50 \%$ stiffness reduction except that error in element 15 is relatively large (only

$720 \%$ stiffness reduction). This can be explained since element 15 is at the edge of the damage

8 area and the vibration energy in this kind of elements would be much more disturbed than

9 those in other elements, which coincides with the observations in literature [35]. The capability

10 of identification was also demonstrated by investigating its convergence behavior during the

11 iterations of damage identification. Figure 9(b) proves that the proposed approach converges

12 to the desirable solution very fast.

13 Table 2 and Figure 10 demonstrate the frequency and mode shape comparisons of the

14 numerical model before (Initial) and after (Updated) identification. As it is observed in Table 2,

15 the reproduction of the frequencies of modes 1-2 and 4-5 have been updated to a better extent.

16 Moreover, the 3th and 6th natural frequencies, which were not involved in the identification 
Table 2 Comparison of frequencies $(\mathrm{Hz})$

\begin{tabular}{ccccccc}
\hline Mode & 1 & 2 & 3 & 4 & 5 & 6 \\
\hline Measured & $2.65^{2}$ & $8.0^{2}$ & $26.56^{1}$ & $45.49^{3}$ & $53.27^{2}$ & $67.36^{1}$ \\
Initial & 2.80 & 8.00 & 26.99 & 46.68 & 55.59 & 70.79 \\
Updated & 2.66 & 8.01 & 26.53 & 46.20 & 53.13 & 67.60 \\
\hline \multicolumn{1}{l}{ not used in damage identification. } \\
2 obtained from accelerometer. \\
3 obtained from vision-based measurement.
\end{tabular}

Table 3 Comparison of MAC

\begin{tabular}{ccccc}
\hline Mode & 1 & 2 & 4 & 5 \\
\hline Initial vs. Measured & 0.9991 & 0.9977 & 0.9696 & 0.9508 \\
Updated vs. Measured & 0.9995 & 0.9978 & 0.9953 & 0.9969 \\
\hline
\end{tabular}

1 process, also got improved significantly.

2 The comparison of mode shapes was further done by calculating the modal assurance cri-

3 teria (MAC) in Table 3. A large MAC value between measured data and numerical data

4 indicates greater correlation in these two mode shapes. The reproduced mode shapes matched

5 the measured data very well, indicating the success of the identification.

6 As mentioned above, the objective function of damage identification is in the weighted least-

7 squares form for which the residual between the measured data and the corresponding analytic

8 data and a weight matrix are involved. Normally, the choice of the weight matrix ( $\boldsymbol{W}$ in Eq. (4))

9 would have a significant effect on the success of the identification results. The optimal weight

10 matrix is found to be inverse proportional to the measurement error covariance by minimizing the

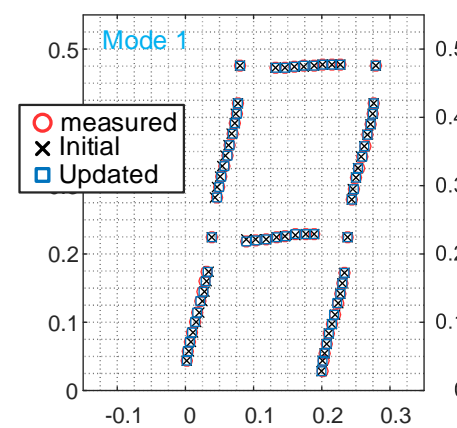

(a)

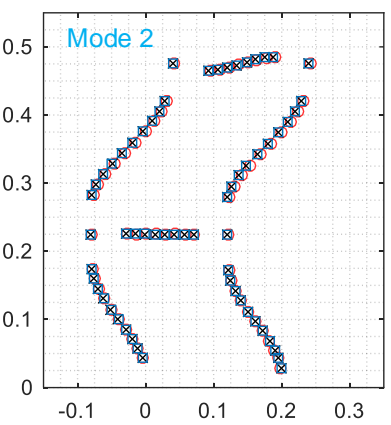

(b)

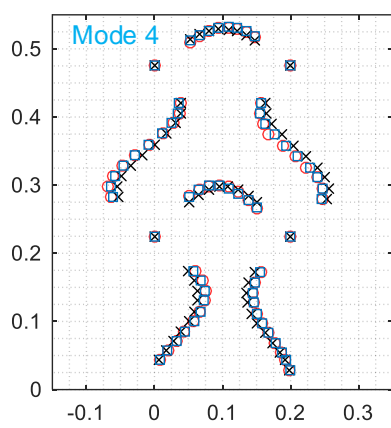

(c)

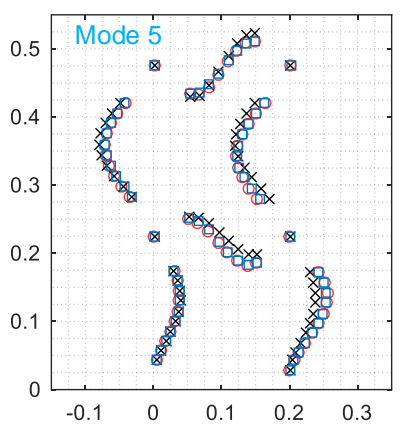

(d)

Figure 10 Measured and numerical mode shapes using vision-based measurements 
Table 4 Different sets of weight matrices considered in the objective function

\begin{tabular}{cccccc}
\hline \multirow{2}{*}{ Scenario } & Weight & \multicolumn{5}{c}{ Mode } \\
\cline { 2 - 6 } & & 1 & 2 & 4 & 5 \\
\hline \multirow{2}{*}{ Optimal weight } & $W_{\lambda_{j}}$ & 1 & 0.5 & 0.1 & 0.4 \\
& $W_{\Phi_{j}}$ & 0.2 & 0.05 & 0.01 & 0.01 \\
& $W_{\lambda_{j}}$ & 1 & 1 & 1 & 1 \\
Identity weight [37] & $W_{\Phi_{j}}$ & 0.01 & 0.01 & 0.01 & 0.01 \\
\hline
\end{tabular}

1 expectation of squares error of the whole identification results [36]. In this research, the relative

2 measurement error of frequency and mode shape is assumed to be independent and follows a

3 normal distribution with zero mean and given variance $\sigma^{2}$, which results in a diagonal optimal

4 weight matrix with different values of main diagonal entries. The variance of the frequency

5 error could be directly obtained from Table 1 and the mode shape counterpart is calculated

6 from the statistic properties in Figure 8. The final optimal weights used in this example

7 have been shown in Table 4. Table 4 also shows the frequently used identity weights (for

8 the same type of measured data) in preliminary damage identification researches [37] and the

9 corresponding identified results are demonstrated in Figure 9(d). It turns out that much less

10 accurate identification results are yielded based on the identity weights. This is due to the

11 above-mentioned different levels of noise in different modes in the camera system. As noise

12 levels for different modes may vary greatly, the weight matrix shall be carefully selected in the

13 sensitivity analysis for vision-based damage identification.

14 To have an impression on the superiority of using vision-based measurement, damage iden-

15 tification using traditional acceleration measurement is further conducted as follows. Nine ac-

16 celerometers (one for ground motion and eight for structural responses) were installed, as shown

17 in Figure 11(a) (blue solid circles). The sampling frequency was set to $200 \mathrm{~Hz}$. Because of the

18 limited number of measurement data obtained in this test, two remedy strategies were employed

19 so as to solve the possible ill-posed problem:

- A numerical model was re-established using much less elements (20 elements to be identified in total) than the one used during vision-based damage identification (63 elements to be identified), as shown in Figure 11(a). In this new model, the damage parameter in element 3 is expected to be identified as $\theta_{3}=0.5$.

- More mode shapes and frequencies were required. Whereas using the acceleration data failed to identify mode 4 of the structure, the frequencies and mode shapes of mode 3 and 


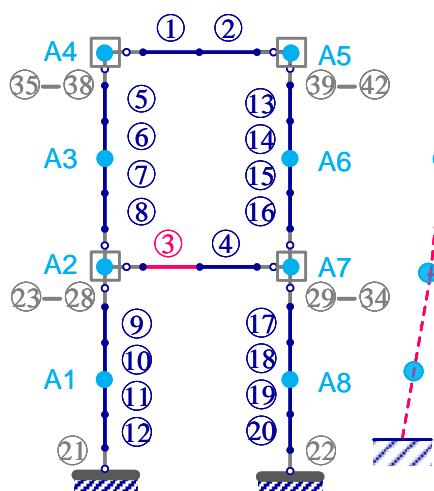

(a)

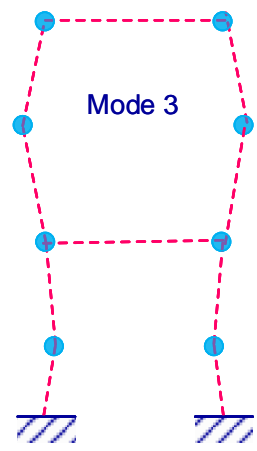

(d)

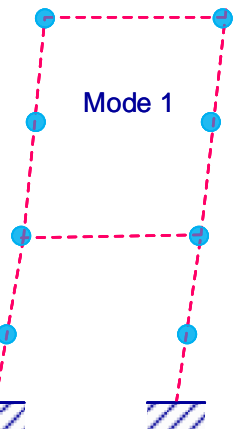

(b)

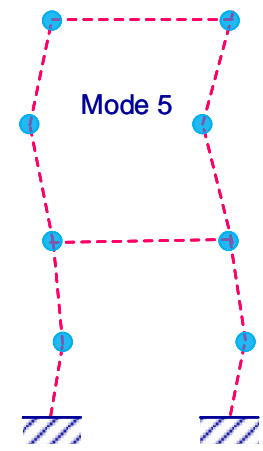

(e)

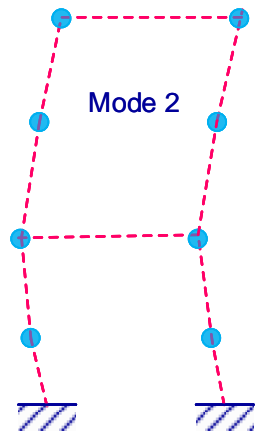

(c)

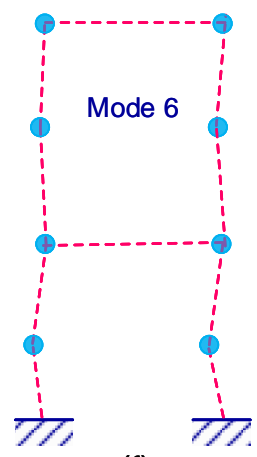

(f)

Figure 11 Numerical model and mode shapes using acceleration measurements: (a) numerical model; (b)-(f) identified mode shapes mode 6 were additionally identified, as shown in Figure 11(b)-(f).

2 The damage identification results by using traditional acceleration measurement data are shown

3 in Figure 12. On can find that although more modes are used and less elements are identified,

4 the identification results are still not as satisfying as that from vision-based measurements. For

5 one thing, the stiffness reduction in the damaged element 3 is not close to $50 \%$ enough; for the

6 other thing, errors in the undamaged elements are large (some equal to 20\%). These observations

7 imply that measurement data is still not sufficient for successful damage identification. Data

8 from more accelerometers or higher modes should be involved. However, this tough situation

9 can be easily circumvented by the vision-based measurement technique.

\section{Discussions}

\section{$11 \quad 5.1 \quad$ Natural frequency measuring accuracy}

12 In section 4.3, it was mentioned that the natural frequencies obtained by the traditional ac13 celerometer are more accurate than those obtained by vision-based measurements because the 


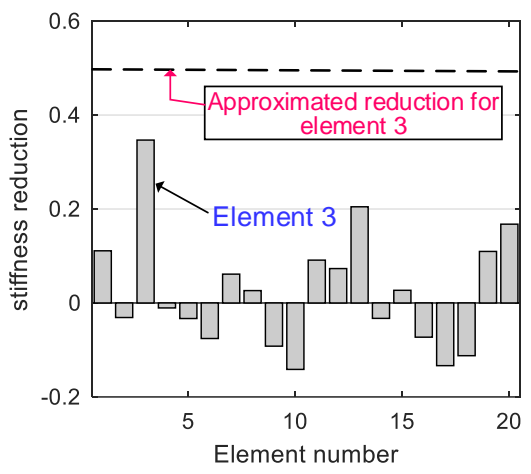

Figure 12 Identification results using traditional accelerometers

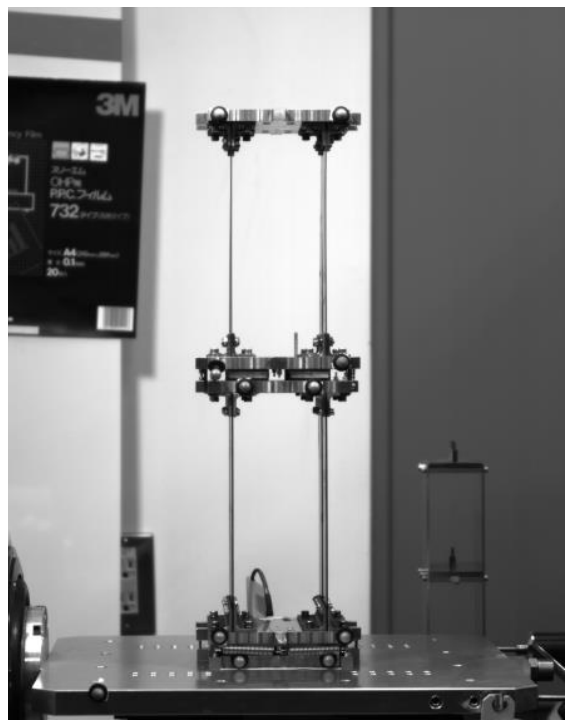

Figure 13 Overview of the two-story isolated based shear building 
Table 5 Measured frequencies of the shear building structure

\begin{tabular}{ccccccc}
\hline Measurement & Mode & Set 1 $(\mathrm{Hz})$ & Set 2 $(\mathrm{Hz})$ & Set 3 $(\mathrm{Hz})$ & Mean $(\mathrm{Hz})$ & $\mathrm{COV}\left(\times 10^{-2}\right)$ \\
\hline \multirow{2}{*}{ Accelerometer } & 1 & 2.744 & 2.741 & 2.740 & 2.74 & 0.062 \\
& 2 & 12.549 & 12.547 & 12.550 & 12.55 & 0.009 \\
\multirow{2}{*}{ Vision-based } & 1 & 2.741 & 2.745 & 2.745 & 2.74 & 0.069 \\
& 2 & 12.543 & 12.541 & 12.550 & 12.54 & 0.031 \\
\hline
\end{tabular}

1 limited camera onboard memory made the vision-based measurement duration very short (only

215 seconds). To compare the natural frequency accuracy using different measurements under the

3 same condition, an additional laboratory test of a two-story base-isolated shear building struc-

4 ture (Figure 13) measured by accelerometers and camera system simultaneously was conducted

5 after the camera onboard memory was expanded. The mass of the isolation story, first story

6 and second story is $2.1 \mathrm{~kg}, 3.247 \mathrm{~kg}$ and $1.531 \mathrm{~kg}$. The stiffness of the spring in the isolation story,

7 first story and second story is about $2.3 \times 10^{3} \mathrm{~N} / \mathrm{m}, 2.3 \times 10^{4} \mathrm{~N} / \mathrm{m}$ and $7.5 \times 10^{3} \mathrm{~N} / \mathrm{m}$, separately.

8 The structure is subjected to a stationary ground excitation of white noise with the duration

9 of two minutes. The sampling rate of the accelerometer in each floor is set to $200 \mathrm{~Hz}$. The

10 setup of the camera system is almost the same as section 4.1, except that the frame rate of the

11 camera is set to $200 \mathrm{fps}$. After three times repeated tests, the mean values of the identified first

12 two natural frequencies with coefficient of variation (COV) of the structure can be obtained, as

13 listed in Table 5. Compared with the results in Table 1, obvious improvements in the natural

14 frequencies obtained by the vision-based measurement can be seen, which verifies the feasibility

15 of the camera system in measuring structural natural frequencies and the suitability in damage

16 identification. To this end, using part of the accelerometer identified natural frequencies in the

17 identification algorithm is unnecessary. Only vision-based measurement is enough.

\section{$18 \quad 5.2 \quad$ Measuring efficiency}

19 In the framework of the proposed method, computation time for each procedure is given as

20 follows: (1) camera calibration: 15 minutes, (2) image processing: 55 minutes, (3) system

21 identification: 58 seconds, and (4) sensitivity analysis: 6.8 seconds. Obviously, the vision-based

22 displacement measurement procedure $(1)+(2)$ leads to the highest computational cost. Some

23 real-time displacement measurement techniques (e.g., $[9,38]$ ) are the possible way to avoid this

24 high computational cost, on the condition that only one target point was selected to be tracked

25 during the field test in reference [38], or low frame rate (30-60 fps) was applied [9]. To measure 
1 the displacements of multiple locations or full-field simultaneously with high frame rate in real

2 time is still a challenging issue.

\section{$3 \quad 5.3 \quad$ Lens distortion}

4 Lens distortion was also considered during the process of camera calibration and the first two

5 terms of radial distortion were estimated, where $k_{1}=-0.2096$ and $k_{2}=0.7519$. As the radial

6 distortion is shown to be small, it is reasonable to simply ignore the undistorition step for

7 computational efficiency. The reader is referred to [39, 40] for details of the distortion model

8 and the solving process for the radial distortion coefficients. Note that for the wide-angle lens

9 or the low-end webcam, the camera usually exhibits significant lens distortion and thus the

10 undistortion step is inevitable.

\section{$11 \quad 5.4 \quad$ Field of view (FOV)}

12 Another challenging issue in full-field vision-based measurement is the tradeoff between the

13 measurement resolution and the field of view (FOV) [41]. A lower measurement resolution and

14 a larger FOV has to be set when measuring multiple points for practical large-scale structures.

15 Such limitations should also be considered for damage identification problems. One possible way

16 to solve this problem is to use multiple synchronized camera, as described in references [42, 43].

\section{$17 \quad 5.5 \quad$ Further research}

18 The proposed approach takes advantage of the rich measured data, which allows considerably 19 greater flexibility for the identified parameters. The current approach in this research is mainly 20 focused on the application with homogeneous conditions. Further research will include the

21 identification of large sets of anisotropic or non-homogeneous constitutive properties.

\section{Conclusions}

23 This paper demonstrated how the newly developed vision-based measurement techniques could

24 be used to localize and quantify structural damages for frame structures. This procedure is 25 composed of four steps: camera calibration using homography estimation with the direct linear 26 transformation (DLT) algorithm, image processing with correlation trackers to extract motions 27 of the selected points through each successive image, system identification by the subspace 28 method and sensitivity analysis for damage identification. Different from the traditional finite29 point measurement techniques, experimental results of a two-story frame structure showed that 
1 vision-based measurement allowed to extract more information from small numbers of test at

2 reasonable levels of accuracy for damage identification problems. Despite the aforementioned

3 advantages, new problem arose when applying this technique. The camera system has been

4 found that it suffered high level of noise in small structural vibration responses at higher fre-

5 quency range. To alleviate this negative effect, the strategy to choose the optimal weights for the

6 sensitivity-based damage identification method was discussed. Further works involves the iden-

7 tification of large sets of more complex constitutive properties for practical large-scale structures

8 using cost-effective vision-based measurement.

\section{Acknowledgement}

The present research was performed under the support of the MEXT scholarship of Japan.

\section{References}

[1] Jia Guo, Li Wang, and Izuru Takewaki. Static damage identification in beams by minimum constitutive relation error. Inverse Problems in Science and Engineering, pages 1-25, 2018.

[2] Stéphane Avril, Marc Bonnet, Anne-Sophie Bretelle, Michel Grédiac, François Hild, Patrick Ienny, Félix Latourte, Didier Lemosse, Stéphane Pagano, Emmanuel Pagnacco, et al. Overview of identification methods of mechanical parameters based on full-field measurements. Experimental Mechanics, 48(4):381, 2008.

[3] Ali Moussawi, Gilles Lubineau, Eric Florentin, and Benoit Blaysat. The constitutive compatibility method for identification of material parameters based on full-field measurements. Computer Methods in Applied Mechanics and Engineering, 265:1-14, 2013.

[4] Dongming Feng and Maria Q Feng. Model updating of railway bridge using in situ dynamic displacement measurement under trainloads. Journal of Bridge Engineering, 20(12):04015019, 2015.

[5] D Ribeiro, R Calçada, J Ferreira, and T Martins. Non-contact measurement of the dynamic displacement of railway bridges using an advanced video-based system. Engineering Structures, 75:164-180, 2014.

[6] James Mark William Brownjohn, Yan Xu, and David Hester. Vision-based bridge deformation monitoring. Frontiers in Built Environment, 3:23, 2017. 
[7] Richard Hartley and Andrew Zisserman. Multiple view geometry in computer vision. Cambridge university press, 2003.

[8] Dongming Feng, Maria Feng, Ekin Ozer, and Yoshio Fukuda. A vision-based sensor for noncontact structural displacement measurement. Sensors, 15(7):16557-16575, 2015.

[9] Dongming Feng and Maria Q Feng. Experimental validation of cost-effective vision-based structural health monitoring. Mechanical Systems and Signal Processing, 88:199-211, 2017.

[10] Li-Jun Wu, Fabio Casciati, and Sara Casciati. Dynamic testing of a laboratory model via vision-based sensing. Engineering structures, 60:113-125, 2014.

[11] Zhengyou Zhang. A flexible new technique for camera calibration. IEEE Transactions on pattern analysis and machine intelligence, 22, 2000.

[12] Zhou Chuan, Tan Da Long, Zhu Feng, and Dong Zai Li. A planar homography estimation method for camera calibration. In Proceedings 2003 IEEE International Symposium on Computational Intelligence in Robotics and Automation. Computational Intelligence in Robotics and Automation for the New Millennium (Cat. No. 03EX694), volume 1, pages 424-429. IEEE, 2003.

[13] Javad Baqersad, Peyman Poozesh, Christopher Niezrecki, and Peter Avitabile. Photogrammetry and optical methods in structural dynamics-a review. Mechanical Systems and Signal Processing, 86:17-34, 2017.

[14] Michel Bornert, Fabrice Brémand, Pascal Doumalin, J-C Dupré, Marina Fazzini, Michel Grediac, François Hild, Sebastien Mistou, Jérôme Molimard, J-J Orteu, et al. Assessment of digital image correlation measurement errors: methodology and results. Experimental mechanics, 49(3):353-370, 2009.

[15] Bing Pan, Anand Asundi, Huimin Xie, and Jianxin Gao. Digital image correlation using iterative least squares and pointwise least squares for displacement field and strain field measurements. Optics and Lasers in Engineering, 47(7-8):865-874, 2009.

[16] Giacomo Lionello and Luca Cristofolini. A practical approach to optimizing the preparation of speckle patterns for digital-image correlation. Measurement Science and Technology, 25(10):107001, 2014. 
[17] Zhenning Chen, Xinxing Shao, Xiangyang Xu, and Xiaoyuan He. Optimized digital speckle patterns for digital image correlation by consideration of both accuracy and efficiency. Applied optics, 57(4):884-893, 2018.

[18] Justin G Chen, Neal Wadhwa, Young-Jin Cha, Frédo Durand, William T Freeman, and Oral Buyukozturk. Modal identification of simple structures with high-speed video using motion magnification. Journal of Sound and Vibration, 345:58-71, 2015.

[19] Hyungchul Yoon, Hazem Elanwar, Hajin Choi, Mani Golparvar-Fard, and Billie F Spencer Jr. Target-free approach for vision-based structural system identification using consumer-grade cameras. Structural Control and Health Monitoring, 23(12):1405-1416, 2016.

[20] C Almeida Santos, C Oliveira Costa, and J Batista. A vision-based system for measuring the displacements of large structures: Simultaneous adaptive calibration and full motion estimation. Mechanical Systems and Signal Processing, 72:678-694, 2016.

[21] Z Hou, M Noori, and R St Amand. Wavelet-based approach for structural damage detection. Journal of Engineering mechanics, 126(7):677-683, 2000.

[22] Zhi-Bo Yang, Maciej Radzienski, Pawel Kudela, and Wieslaw Ostachowicz. Fourier spectralbased modal curvature analysis and its application to damage detection in beams. Mechanical Systems and Signal Processing, 84:763-781, 2017.

[23] Michael Friswell and John E Mottershead. Finite element model updating in structural dynamics, volume 38. Springer Science \& Business Media, 2013.

[24] John E Mottershead, Michael Link, and Michael I Friswell. The sensitivity method in finite element model updating: a tutorial. Mechanical Systems and Signal Processing, 25(7):2275$2296,2011$.

[25] Edward Sazonov and Powsiri Klinkhachorn. Optimal spatial sampling interval for damage detection by curvature or strain energy mode shapes. Journal of sound and vibration, 285(4-5):783-801, 2005.

[26] Lorenzo Montanari, Andrea Spagnoli, Biswajit Basu, and Brian Broderick. On the effect of spatial sampling in damage detection of cracked beams by continuous wavelet transform. Journal of Sound and Vibration, 345:233-249, 2015. 
[27] M Mininni, S Gabriele, H Lopes, and JV Araújo dos Santos. Damage identification in beams using speckle shearography and an optimal spatial sampling. Mechanical Systems and Signal Processing, 79:47-64, 2016.

[28] JE Mottershead, C Mares, MI Friswell, and S James. Selection and updating of parameters for an aluminium space-frame model. Mechanical Systems and Signal Processing, 14(6):923$944,2000$.

[29] Elan Dubrofsky. Homography estimation. Diplomová práce. Vancouver: Univerzita Britské Kolumbie, 2009.

[30] CD Zhang and YL Xu. Comparative studies on damage identification with tikhonov regularization and sparse regularization. Structural Control and Health Monitoring, 23(3):560-579, 2016.

[31] Jia Guo, Kailai Deng, Li Wang, and Izuru Takewaki. Physical-based parametrization and local damage identification for frame-type structures using response sensitivity approach in time domain. Structural Control and Health Monitoring, page e2412.

[32] Jia Guo, Li Wang, and Izuru Takewaki. Frequency response-based damage identification in frames by minimum constitutive relation error and sparse regularization. Journal of Sound and Vibration, 443:270-292, 2019.

[33] Eric M Hernandez. Identification of isolated structural damage from incomplete spectrum information using 11-norm minimization. Mechanical Systems and Signal Processing, 46(1):59-69, 2014.

[34] Richard B Nelson. Simplified calculation of eigenvector derivatives. AIAA journal, 14(9):1201-1205, 1976.

[35] ZY Shi, SS Law, and LM Zhang. Structural damage detection from modal strain energy change. Journal of Engineering Mechanics-ASCE, 126(12):1216-1223, 2000.

[36] Zhong-Rong Lu, Junxian Zhou, and Li Wang. On choice and effect of weight matrix for response sensitivity-based damage identification with measurement and model errors. Mechanical Systems and Signal Processing, 114:1-24, 2019.

[37] Hong Hao and Yong Xia. Vibration-based damage detection of structures by genetic algorithm. Journal of computing in civil engineering, 16(3):222-229, 2002. 
[38] Jong-Jae Lee and Masanobu Shinozuka. Real-time displacement measurement of a flexible bridge using digital image processing techniques. Experimental mechanics, 46(1):105-114, 2006 .

[39] C Brown Duane. Close-range camera calibration. Photogramm. Eng, 37(8):855-866, 1971.

[40] YM Wang, Y Li, and JB Zheng. A camera calibration technique based on opencv. In The 3rd International Conference on Information Sciences and Interaction Sciences, pages 403-406. IEEE, 2010.

[41] Dongming Feng and Maria Q Feng. Computer vision for shm of civil infrastructure: From dynamic response measurement to damage detection-a review. Engineering Structures, 156:105-117, 2018.

[42] Jong-Han Lee, Hoai-Nam Ho, Masanobu Shinozuka, and Jong-Jae Lee. An advanced visionbased system for real-time displacement measurement of high-rise buildings. Smart Materials and Structures, 21(12):125019, 2012.

[43] Yoshio Fukuda, Maria Q Feng, and Masanobu Shinozuka. Cost-effective vision-based system for monitoring dynamic response of civil engineering structures. Structural Control and Health Monitoring, 17(8):918-936, 2010. 